\title{
Development of a 2-Layer Double-Pump Dynamic Cardiac Phantom
}

\author{
Hara Narihiro $^{1,2}$, Onoguchi Masahisa ${ }^{2}$, Hojyo Osamu $^{1}$, Kawaguchi Hiroyuki $^{1}$, Murai Masakazu ${ }^{1}$, and Matsushima Noriko ${ }^{1}$

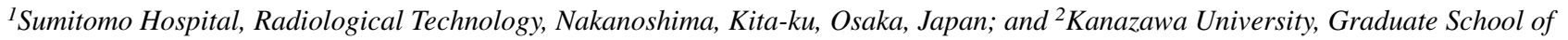 \\ Medical Sciences, Kodatsuno, Kanazawa, Japan
}

The conventional dynamic cardiac phantom used in the field of nuclear medicine has a structure for which the size of the external side of the heart (the outer membrane substituting the myocardial layer) is fixed and only the inner side (the inner membrane substituting the ventricle part) moves anteroposteriorly. Therefore, its usefulness in technical evaluation is limited. Hence, we developed a new dynamic cardiac phantom in which the outer and inner membranes freely move. Methods: Using a SPECT/CT system, we performed validation by filling the myocardial layer of the dynamic cardiac phantom with solution and the ventricle part with contrast medium. We evaluated myocardial wall motions of 3 segments (basal, mid, and apical) by setting the stroke ratios at 20:20 and 10:10 (ventricle-to-myocardial layer ratio). Results: The myocardial wall motions (mean $\pm \mathrm{SD}$ ) at the stroke ratio of $20: 20$ were $7.50 \pm 0.44,11.15 \pm 0.56$, and $9.90 \pm 0.24 \mathrm{~mm}$ in the basal, mid, and apical segments, respectively. The wall motions (mean \pm SD) at the stroke ratio of $10: 10$ were $3.82 \pm 0.43,5.63 \pm 0.39$, and $4.53 \pm 0.10 \mathrm{~mm}$, respectively. Conclusion: In our dynamic cardiac phantom, different movements could be induced in the myocardial wall by freely changing the stroke ratio. These results suggest that the use of this phantom can realize technical evaluation that presumes various clinical conditions.

Key Words: dynamic cardiac phantom; myocardial wall movement; gated myocardial SPECT; SPECT/CT

J Nucl Med Technol 2016; 44:31-35

DOI: 10.2967/jnmt.115.168252

$\mathbf{N}$ uclear medicine examination using gated myocardial SPECT can provide image information of high clinical usefulness as a functional diagnostic method (1-5) and much evidence for the diagnosis of ischemic heart disease $(6-8)$, assessment of severity $(9,10)$, determination of treatment efficacy $(11,12)$, prediction of prognosis, and stratification of risk (13-15). To secure and establish evidence, technical evaluation is needed as a basic study.

\footnotetext{
Received Oct. 16, 2015; revision accepted Dec. 28, 2015.

For correspondence or reprints contact: Hara Narihiro, Sumitomo Hospital,

5-3-20, Nakanoshima, Kita-ku, Osaka, 530-0005, Japan.

E-mail: hara-narihiro@sumitomo-hp.or.jp

Published online Jan. 14, 2016.

COPYRIGHT (C 2016 by the Society of Nuclear Medicine and Molecular Imaging, Inc.
}

However, the conventional dynamic cardiac phantom used for technical evaluation of gated myocardial SPECT (16) has a structure for which the size of the external side of the heart (the outer membrane substituting the myocardial layer) is fixed and only the inner side (the inner membrane substituting the ventricle) moves anteroposteriorly (Fig. 1A). Therefore, detailed technical evaluation corresponding to actual clinical conditions cannot be performed. To perform technical evaluation toward clinical application, we developed a new dynamic cardiac phantom in which the outer and inner membranes move freely (Figs. 1B and 2A). The myocardial layer comprises outer and inner membranes made of special rubber, and heart movement is reproduced using 2 cylinder pumps (Fig. 2B). The outlet (discharge outlet, 15) for solutions supplied to the area of the ventricle and myocardial layer (e.g., radioactive and contrast agents) has a special structure (Fig. 2C). The contents of the ventricle and myocardial layer can be changed by setting the stroke ratio of the cylinder pumps freely (Fig. 2D).

\section{MATERIALS AND METHODS}

\section{Setting of Phantom}

The movable parts of the myocardial layer and ventricle in the new dynamic cardiac phantom were filled with a mixed solution of radioactive agent $(755.00 \mathrm{~mL}$ of distilled water and $0.74 \mathrm{MBq}$ of ${ }^{99 \mathrm{~m}} \mathrm{TC}$ solution per $\left.\mathrm{mL}\right)$ and contrast agent $(982.00 \mathrm{~mL}$ of distilled water and $370 \mathrm{mg}$ of iodine concentration per $\mathrm{mL}$ ), respectively. Table 1 shows the actual measurement values calculated from the structure of the new dynamic cardiac phantom (end-diastolic volume [EDV], end-systolic volume [ESV], and left ventricular ejection fraction [LVEF]).

At the time of SPECT data acquisition, the heart rate was fixed at $60 \mathrm{bpm}$, and 11 patterns of stroke ratio (ventricle-to-myocardial layer ratio) were set (20:20, 20:10, 20:5, 15:15, 15:10, 15:5, 10:10, $10: 5,10: 20,10: 15$, and 5:10).

\section{Gated SPECT Data Acquisition and Image Reconstruction}

A SPECT/CT system (Precedence 16; Philips) was used. The conditions for gated SPECT data acquisition were data acquisition time at $30 \mathrm{~s} / \mathrm{step}\left(360^{\circ}, 64\right.$ steps), R-R interval at 16 , use of a vertex general-purpose collimator, and matrix sizes of $128 \times 128$ (pixel size, $3.19 \mathrm{~mm}$ ) and $64 \times 64(6.39 \mathrm{~mm})$ (concurrent imaging: different acquisition data can be stored at 1 imaging). 
FIGURE 1. (A) Schematic diagram of conventional dynamic cardiac phantom for nuclear medicine. (B) Schematic diagram of new dynamic cardiac phantom for nuclear medicine.

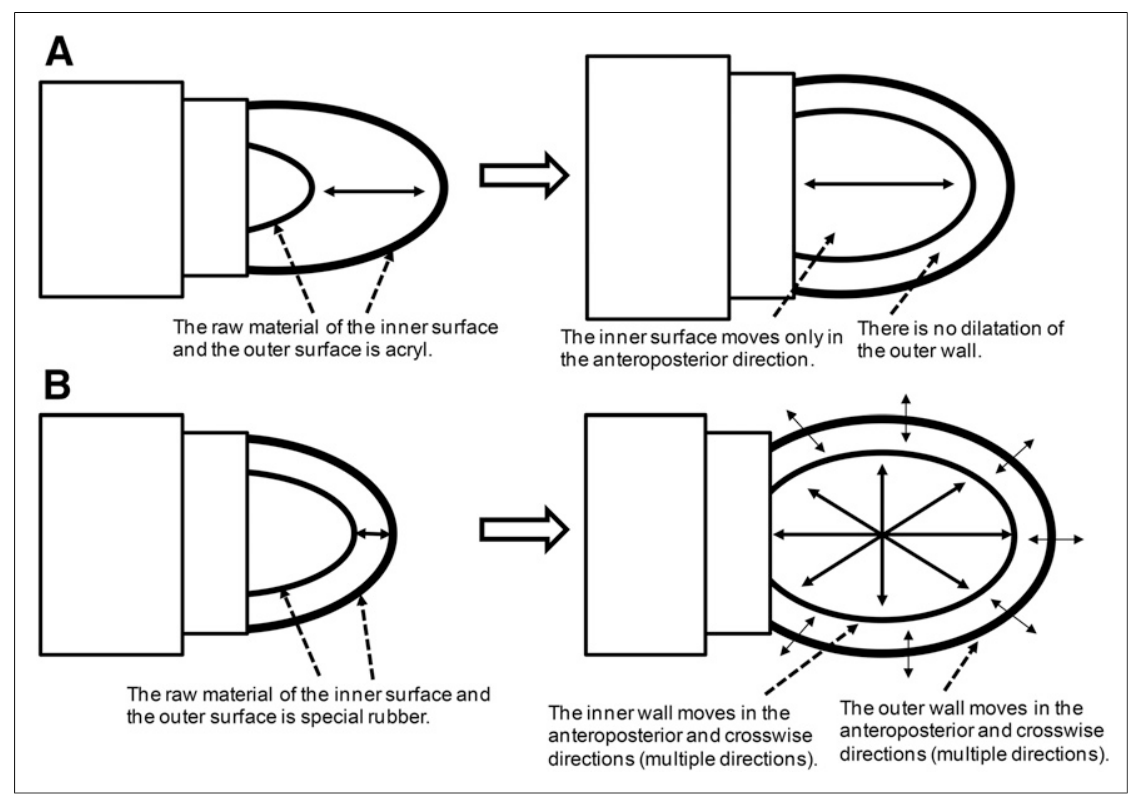

Gated myocardial perfusion image reconstruction was performed using filtered backprojection (cutoff of 0.19 cycles/cm and order of 9 for a matrix size of $128 \times 128$ ) and a Butterworth filter for the preprocessing filter (cutoff of 0.37 cycles/cm and order of 9 for a matrix size of $64 \times 64)$. A ramp filter was used as the reconstruction filter, without attenuation correction and scatter correction.

\section{Analysis}

Results of the cardiac function analysis of the SPECT data (EDV, ESV, LVEF, myocardial wall motion, and changes in wall thickness) were processed using EBW-NM4.5 (Philips). The Statistical Package for Social Sciences software (IBM) was used for the statistical analysis.

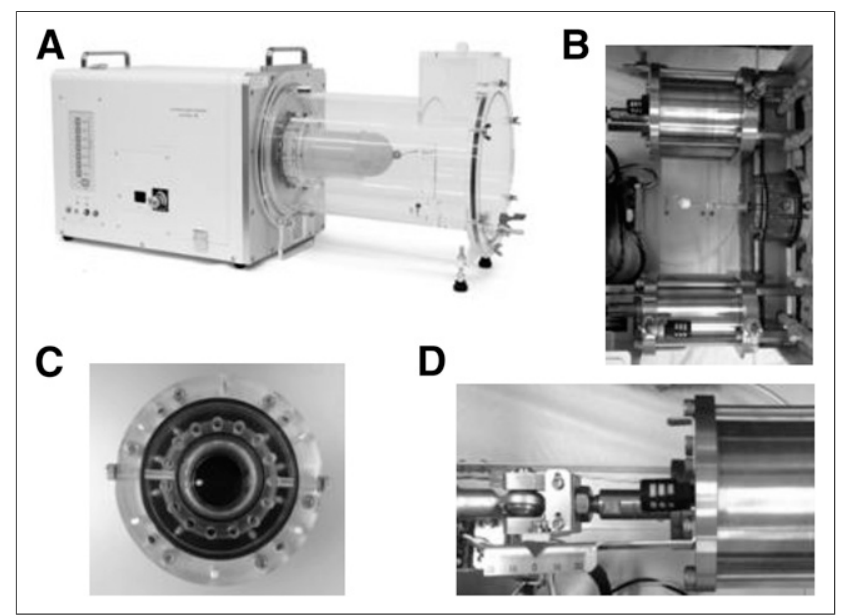

FIGURE 2. (A) Our new dynamic cardiac phantom. (B) Appearance of 2 cylinder pumps. (C) Appearance of outlet (front). (D) Scale of stroke.

\section{RESULTS}

Table 1 shows the actual measurement values obtained using our new dynamic cardiac phantom and the EDV, ESV, and LVEF analyzed from the SPECT images. Figure 3 shows the results of the statistical analysis. In the comparison between the actual measurement values and the SPECT analysis values, high correlations were observed for a matrix size of 128 (EDV, $r=0.92$; ESV, $r=0.93$; LVEF, $r=0.95$ ) and a matrix size of 64 (EDV, $r=0.90$; ESV, $r=0.88$; LVEF, $r=0.95$ ).

Figure 4 shows the results of the myocardial wall motion at each stroke ratio. The wall motions at each stroke ratio were within the ranges of 2.13-7.50, 3.32-11.10, and $3.00-9.90 \mathrm{~mm}$ in the basal, mid, and apical segments, respectively, for a matrix size of 128, and 2.10-7.87, $3.23-11.27$, and $3.05-10.25 \mathrm{~mm}$, respectively, for a matrix size of 64 (Fig. 4).

Figure 5 shows the results of the changes in wall thickness at each stroke ratio. The maximum and minimum changes in wall thickness at each stroke ratio (mean \pm SD) for a matrix size of 128 were $16.55 \pm 1.56$ and $5.47 \pm 0.64 \mathrm{~mm}, 11.35 \pm$ 3.29 and $2.45 \pm 0.85 \mathrm{~mm}$, and $9.73 \pm 2.91$ and $2.75 \pm$ $1.48 \mathrm{~mm}$ in the basal, mid, and apical segments, respectively, and those for a matrix size of 64 were $17.90 \pm 1.66$ and $5.87 \pm$ $0.65 \mathrm{~mm}, 12.83 \pm 3.49$ and $2.67 \pm 1.03 \mathrm{~mm}$, and $10.90 \pm 3.14$ and $2.88 \pm 1.45 \mathrm{~mm}$, respectively (Fig. 5).

\section{DISCUSSION}

The usefulness of gated myocardial SPECT in the quantitative evaluation of transient left ventricular dysfunction and myocardial blood flow impairment has been reported, and it has improved the accuracy of evaluations of severity $(9,10)$, risk, and prognosis $(13-15)$. In addi- 
TABLE 1

Actual Measurement Values and SPECT Analysis Values (EDV, ESV, and LVEF) at Different Stroke Ratios

\begin{tabular}{|c|c|c|c|c|c|c|c|c|c|}
\hline \multirow{3}{*}{$\begin{array}{c}\text { Stroke } \\
\text { rate }\end{array}$} & \multicolumn{3}{|c|}{ EDV (mL) } & \multicolumn{3}{|c|}{ ESV (mL) } & \multicolumn{3}{|c|}{ LVEF (\%) } \\
\hline & \multirow{2}{*}{$\begin{array}{c}\text { Actual } \\
\text { measurement } \\
\text { value }\end{array}$} & \multicolumn{2}{|c|}{ SPECT } & \multirow{2}{*}{$\begin{array}{c}\text { Actual } \\
\text { measurement } \\
\text { value }\end{array}$} & \multicolumn{2}{|c|}{ SPECT } & \multirow{2}{*}{$\begin{array}{c}\text { Actual } \\
\text { measurement } \\
\text { value }\end{array}$} & \multicolumn{2}{|c|}{ SPECT } \\
\hline & & $128 \times 128$ & $64 \times 64$ & & $128 \times 128$ & $64 \times 64$ & & $128 \times 128$ & $64 \times 64$ \\
\hline $20: 20$ & 495.76 & 506.00 & 489.00 & 294.80 & 276.00 & 257.00 & 40.60 & 45.50 & 47.40 \\
\hline $20: 10$ & 495.76 & 473.00 & 465.00 & 294.80 & 271.00 & 262.00 & 40.60 & 42.70 & 43.70 \\
\hline $20: 5$ & 495.76 & 468.00 & 450.00 & 294.80 & 286.00 & 269.00 & 40.60 & 38.90 & 40.20 \\
\hline $15: 15$ & 470.64 & 459.00 & 430.00 & 319.90 & 289.00 & 264.00 & 32.03 & 37.00 & 38.60 \\
\hline $15: 10$ & 470.64 & 451.00 & 438.00 & 319.90 & 292.00 & 285.00 & 32.03 & 35.30 & 34.90 \\
\hline $15: 5$ & 470.64 & 445.00 & 414.00 & 319.90 & 303.00 & 270.00 & 32.03 & 31.90 & 34.80 \\
\hline $10: 10$ & 445.52 & 430.00 & 416.00 & 345.00 & 319.00 & 304.00 & 22.56 & 25.80 & 26.90 \\
\hline $10: 5$ & 445.52 & 425.00 & 410.00 & 345.00 & 333.00 & 317.00 & 22.56 & 21.60 & 22.70 \\
\hline 10:20 & 445.52 & 440.00 & 416.00 & 345.00 & 311.00 & 282.00 & 22.56 & 29.30 & 32.20 \\
\hline $10: 15$ & 445.52 & 432.00 & 421.00 & 345.00 & 306.00 & 297.00 & 22.56 & 29.20 & 29.50 \\
\hline $5: 10$ & 420.40 & 404.00 & 385.00 & 370.16 & 336.00 & 321.00 & 11.95 & 16.80 & 16.60 \\
\hline
\end{tabular}
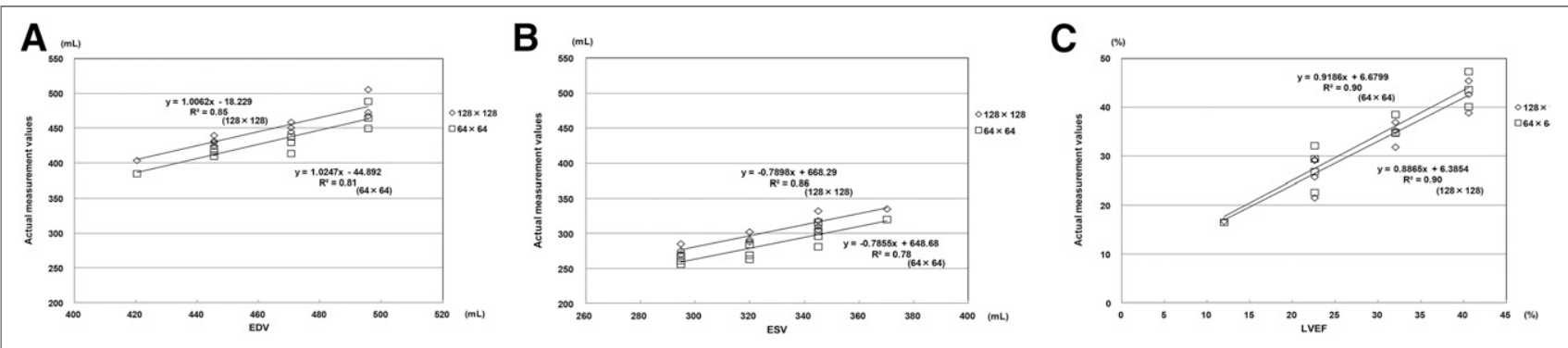

FIGURE 3. Results of statistical analysis of actual measurement values and SPECT analysis values. (A) EDV. (B) ESV. (C) LVEF.

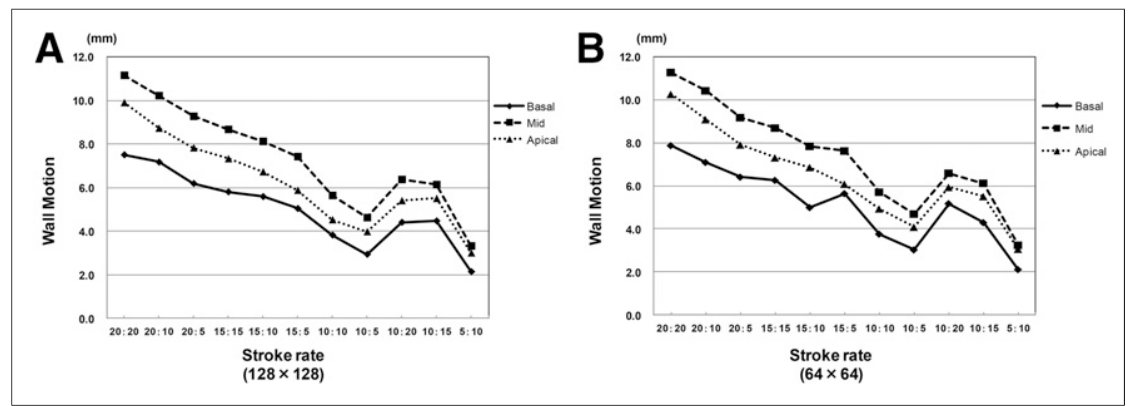

FIGURE 4. Results of stroke ratios and wall motion. (A) Matrix size, $128 \times 128$. (B) Matrix size, $64 \times 64$. tion, gated myocardial SPECT has been reported to be useful for the evaluation of EDV, ESV, LVEF, stroke volume/cardiac index, myocardial wall motion, and changes in myocardial wall thickness, which are critical in risk and prognosis assessment in coronary artery diseases $(1,2)$

Published reports have stated that application of the exercise or drug-loading method by using gated myocardial SPECT can quantitatively evaluate markers of poor prognosis, transient reduction in LVEF after loading, and myocardial wall motion abnormality $(3,4)$. These reports suggest the need of further improvement of the technical accuracy of nuclear cardiology examinations.

Recent advances have led to the development of nuclear cardiology devices mounted with a semiconductor detector, and technical evaluation was performed on these devices (17-19). However, the phantoms used in the previous studies were static cardiac phantoms (17) and cardiac SPECT phantoms (19). With these phantoms, detailed technical evaluation with gated myocardial SPECT cannot be performed. We developed a new dynamic cardiac phantom 
FIGURE 5. Results of stroke ratios and changes in myocardial wall thickness. (A) Matrix size, $128 \times 128$. (B) Matrix size, $64 \times 64$.

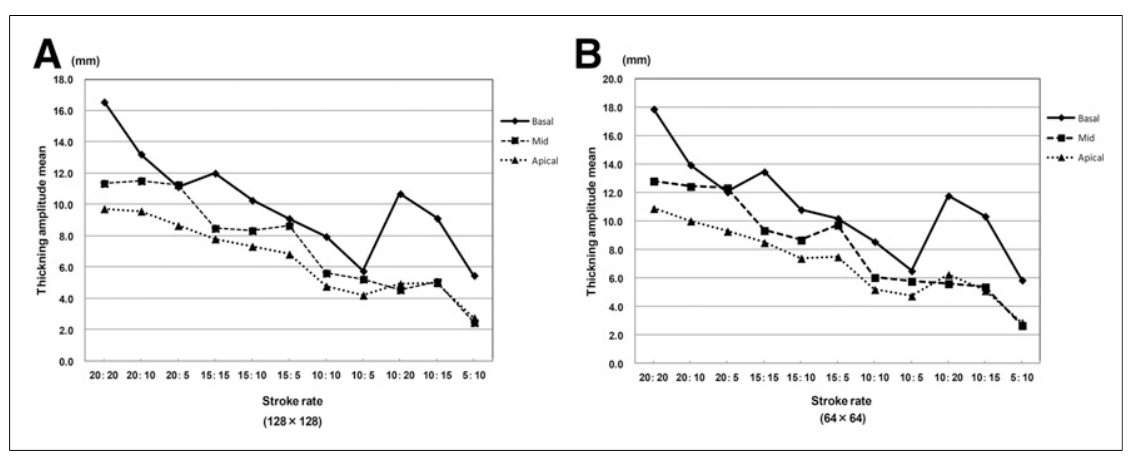

because the evaluation of myocardial wall motion is considered necessary, in addition to the evaluation of EDV, ESV, and LVEF, to perform technical evaluation with high clinical applicability in the future.

Detailed cardiac function analysis cannot be performed with static cardiac phantoms used for technical evaluation in nuclear cardiology examinations (20-22) or the conventional dynamic cardiac phantom developed by Kubo et al. (16), in which cardiac function analytic values (EDV, ESV, and LVEF) are fixed values calculated from the forms of the phantoms. However, the actual measurement values at the different stroke ratios of our new dynamic cardiac phantom showed high correlation with SPECT analysis values, suggesting that detailed validation of cardiac function analysis can be performed by setting stroke ratios appropriate for clinical application (Table 1; Fig. 5).

The dynamic myocardial phantoms reported by Jacco et al. (23) and Luca et al. (24) have 2 discharge outlets for solutions supplied to the myocardial layer, and the same pump is used to supply solutions to the ventricle part and myocardial layer. Although EDV, ESV, and LVEF were evaluated, no detailed evaluation was performed such as that for myocardial wall motion. In our new dynamic cardiac phantom, the solution supply system to the myocardial layer has a special structure (Fig. 2C; discharge outlet, 15). It has independent supply pumps to the ventricle and myocardial layer (Fig. 2B), and stroke ratios can be set freely (Fig. 2D). In addition to the evaluation of EDV, ESV, and LVEF at different stroke ratios (Fig. 3), various myocardial wall motions can be set through the combination of stroke ratios of the cylinder pumps of the ventricle and myocardial layer (Fig. 4). Among the stroke ratios validated, we could confirm free changes in myocardial wall thickness based on the differences between 20:10 and 10:20 (2.54, 6.97, and $4.63 \mathrm{~mm}$ in the basal, mid, and apical segments, respectively), between 15:10 and $10: 15(1.15,3.26$, and $2.33 \mathrm{~mm}$, respectively), and between 10:5 and 5:10 $(0.28,2.78$, and $1.45 \mathrm{~mm}$, respectively (Fig. 5).

\section{CONCLUSION}

This study demonstrates that our new dynamic cardiac phantom can be used for technical evaluation that presumes various clinical conditions. Technical validation for SPECT image processing and attenuation correction using this phantom is needed in the future.

\section{DISCLOSURE}

No potential conflict of interest relevant to this article was reported.

\section{ACKNOWLEDGMENTS}

We thank Munetomo Sato (Fuyo Corporation) and the staff of the Department of Pharmacy of Sumitomo Hospital for their cooperation in this study.

\section{REFERENCES}

1. Nakajima K, Kusuoka H, Nishimura S, et al. Normal limits of ejection fraction and volumes determined by gated SPECT in clinically normal patients without cardiac events: a study based on the J-ACCESS database. Eur J Nucl Med Mol Imaging. 2007;34:1088-1096.

2. Nakajima K, Nishimura T. Inter-institution preference-based variability of ejection fraction and volumes using quantitative gated SPECT with ${ }^{99 \mathrm{~m} T c-t e t r o f o s m i n: ~ a ~}$ multicentre study involving 106 hospitals. Eur J Nucl Med Mol Imaging. 2006;33:127-133.

3. Kapetanopoulos, Ahlberg AW, Taub CC, et al. Regional wall-motion abnormalities on post-stress electrocardiographic-gated technetium- $99 \mathrm{~m}$ sestamibi singlephoton emission computed tomography imaging predict cardiac events. J Nucl Cardiol. 2007;14:810-817.

4. Sharir T. Role of regional myocardial dysfunction by gated myocardial perfusion SPECT in the prognostic evaluation of patients with coronary artery disease. $J$ Nucl Cardiol. 2005;12:5-8.

5. Schaefer WM, Lipke CS, Dirk Standke, et al. Quantification of left ventricular volumes and ejection fraction from gated ${ }^{99 \mathrm{~m}} \mathrm{Tc}-\mathrm{MIBI}$ SPECT: MRI validation and comparison of the Emory cardiac tool box with QGS and 4D-MSPECT. $J$ Nucl Med. 2005;46:1256-1263.

6. Kuwabara Y, Watanabe S, Nakaya J, et al. Functional evaluation of myocardial viability by ${ }^{99 \mathrm{~m}} \mathrm{Tc}$ tetrofosmin gated SPECT: a quantitative comparison with ${ }^{18} \mathrm{~F}$ fluorodeoxyglucose positron emission CT ( ${ }^{18} \mathrm{~F}$ FDG PET). Ann Nucl Med. 1999; 13:135-140.

7. Levine MG, McGill CC, Ahlberg AW, et al. Functional assessment with electrocardiographic gated single-photon emission computed tomography improves the ability of technetium-99m sestamibi myocardial perfusion imaging to predict myocardial viability in patients undergoing revascularization. Am J Cardiol. 1999;83:1-5.

8. Pagnanelli RA, Hanson MW, Turkington T, Coleman RE, Borges-Neto S. Gated ${ }^{99 \mathrm{~m}} \mathrm{Tc}$-tetrofosmin and ${ }^{18} \mathrm{~F}$-FDG studies: a comparison of single-acquisition and separate-acquisition protocols. J Nucl Med Technol. 2002;30:175-178.

9. Slomka PJ, Nishina H, Berman DS, et al. Automated quantification of myocardial perfusion SPECT using simplified normal limits. J Nucl Cardiol. 2005; 12:66-77

10. Berman DS, Kang X, Gransar H, et al. Quantitative assessment of myocardial perfusion abnormality on SPECT myocardial perfusion imaging is more reproducible than expert visual analysis. J Nucl Cardiol. 2009;16:45-53. 
11. Nakajima K, Taki J, Higuchi T, et al. Gated SPET quantification of small hearts: mathematical simulation and clinical application. Eur J Nucl Med. 2000;27:1372-1379.

12. Emre A, Ersek B, Gürsürer M, et al. Angiographic and scintigraphic (perfusion and electrocardiogram-gated SPECT) correlates of clinical presentation in unstable angina. Clin Cardiol. 2000;23:495-500.

13. Matsumoto N, Sato Y, Suzuki Y, et al. Incremental prognostic value of cardiac function assessed by ECG-gated myocardial perfusion SPECT for the prediction of future acute coronary syndrome. Circ J. 2008;72:2035-2039.

14. Petix NR, Sestini S, Coppola A, et al. Prognostic value of combined perfusion and function by stress technetium-99m sestamibi gated SPECT myocardial perfusion imaging in patients with suspected or known coronary artery disease. Am J Cardiol. 2005;95:1351-1357.

15. Travin MI, Heller GV, Johnson LL, et al. The prognostic value of ECG-gated SPECT imaging in patients undergoing stress Tc-99m sestamibi myocardial perfusion imaging. J Nucl Cardiol. 2004;11:253-262.

16. Kubo N, Mabuchi M, Katoh C, et al. Validation of left ventricular function from gated single photon computed emission tomography by using a scintillatorphotodiode camera: a dynamic myocardial phantom study. Nucl Med Commun. 2002;23:639-643.

17. Bocher M, Blevis IM, Tsukerman L, et al. A fast cardiac gamma camera with dynamic SPECT capabilities: design, system validation and future potential. Eur J Nucl Med Mol Imaging. 2010;37:1887-1902.
18. Kacperski K, Erlandsson K, Haim SB, et al. Iterative deconvolution of simultaneous ${ }^{99 \mathrm{~m}} \mathrm{Tc}$ and ${ }^{201} \mathrm{Tl}$ projection data measured on a $\mathrm{CdZnTe}$ based cardiac SPECT scanner. Phys Med Biol. 2011;56:1397-1414.

19. Takahashi Y, Miyagawa M, Nishiyama Y, et al. Performance of a semiconductor SPECT system: comparison with a conventional Anger-type SPECT instrument. Ann Nucl Med. 2013;27:11-16.

20. Beach RD, Depold H, Boening G, et al. An adaptive approach to decomposing patient-motion tracking data acquired during cardiac SPECT imaging. IEEE Trans Nucl Sci. 2007;54:130-139.

21. Knesaurek K, Machac J. Comparison of ${ }^{18} \mathrm{~F}$ SPECT with PET in myocardial imaging: a realistic thorax cardiac phantom study. BMC Nucl Med. 2006; 6:5-13.

22. Difilippo FP. Design and performance of a multi-pinhole collimation device for small animal imaging with clinical SPECT and SPECT-CT scanners. Phys Med Biol. 2008;53:4185-4201.

23. Visser JJ, Sokole EB, Verberne HJ, et al. A realistic 3-D gated cardiac phantom for quality control of gated myocardial perfusion SPET: the Amsterdam gated (AGATE) cardiac phantom. Eur J Nucl Med Mol Imaging. 2004;31:222228.

24. Ceriani L, Ruberto T, Delaloye AB, Prior JO, Giovanella L. Three-dimensional ordered-subset expectation maximization iterative protocol for evaluation of left ventricular volumes and function by quantitative gated SPECT: a dynamic phantom study. J Nucl Med Technol. 2010;38:18-23. 